\title{
NEW METHOD FOR MONITORING THE FUNCTIONAL STATE OF A DYNAMIC CARDIOMYOPLASTY
}

Norbert W. Guldner, MD ${ }^{\mathrm{a}}$

Peter Klapproth, $\mathrm{MSc}^{\mathrm{a}}$

J. Michael Hasenkam, MD, DMSc ${ }^{\mathrm{b}}$

Thomas Fischer, MD ${ }^{\mathrm{c}}$

Robert Keller, MD ${ }^{a}$

Ralf Noel, VD ${ }^{\mathrm{d}}$

Britta Keding, MT

Elrina Joubert-Hübner, $\mathrm{MSc}^{\mathrm{a}}$

Hermann Kuppe, MD, $\mathrm{PhD}^{\mathrm{c}}$

Hans-H. Sievers, MD, $\mathrm{PhD}^{\mathrm{a}}$
Objective: To assess the impact of a dynamic cardiomyoplasty on failing hearts, it is essential to estimate the contraction force of the skeletal muscle and how its contraction is synchronized with the heart cycle. Methods: In a 6-month study a small fluid-filled, balloon-mounted catheter was placed between the myocardium and the muscular wrap in five adult female Boor goats and two female domestic pigs. The catheter was connected to a subcutaneous measuring chamber whereby pressure monitoring could be accomplished. Distinct pressure signals as a result of function of the dynamic cardiomyoplasty and the heart were detected initially in all animals. Results: Maximal relative pressure from the dynamic cardiomyoplasty was calculated as $336.2 \% \pm 69.4 \%$ on day $24 \pm$ 6.1 $(n=7)$ and end-stage pressure as $59.8 \% \pm 9.7 \%$ on day $174.6 \pm 13.1$ $(n=4)$. A functional loss of pressure signals from the dynamic cardiomyoplasty was correlated to severe histologic muscle damage $(n=$ 3). Pressure signals transferred from the contracting myocardium to the catheter showed defined segments of contraction, ejection, and filling periods, allowing a mechanical synchronization of the dynamic cardiomyoplasty to the heart cycle. Conclusions: This monitoring catheter enabled the assessment of the functional state of the dynamic cardiomyoplasty and allowed a synchronization to the heart cycle. It will promote understanding and might help to avoid muscle damage in dynamic cardiomyoplasty for an improved outcome of the surgical treatment of end-stage heart failure. (J Thorac Cardiovasc Surg 1997; 114:1097-106)
Thesente he first application of dynamic cardiomyoplasty (DCMP) was performed in $1985 .{ }^{1}$ Although more than 600 procedures have been performed worldwide since then, the fundamental mechanics of the method are still not understood. Generally the

From the Clinic of Cardiac Surgery, ${ }^{a}$ Institute of Anaesthesiology, ${ }^{\mathrm{c}}$ Department of Experimental Animal Care, ${ }^{\mathrm{d}}$ Medical University of Lübeck, Germany, and from the Department of Cardio-Thoracic and Vascular Surgery, Skejby Sygehus, Aarhus University Hospital, ${ }^{\mathrm{b}}$ Aarhus, Denmark.

Supported by Bakken Research Center, Division of Medtronic, Maastricht, The Netherlands, Telectronics Pacing Systerns Inc., Englewood, Colo., and A.D. Krauth GmbH, Hamburg, Germany.

Received for publication March 3, 1997; revisions requested May 8, 1997; revisions received June 6, 1997; accepted for publication July 30, 1997.

Address for reprints: Norbert W. Guldner, MD, Klinik für Herzchirurgie, Medizinische Universität zu Lübeck, Ratzeburgerallee 160, D-23538 Lübeck, Germany.

Copyright (c) 1997 by Mosby-Year Book, Inc.

$0022-5223 / 97 \$ 5.00+0 \quad \mathbf{1 2 / 1 / 8 5 0 4 3}$ patients seem to have improved physical capacity but objective measures do not always reflect this improvement. ${ }^{2-6}$ In some clinical cases stroke work and left ventricular volume evaluation by the conductance catheter method disclosed functional improvement. ${ }^{7,8}$ This technique only allows occasional assessment and cannot be used for a frequently applied routine examination of the training procedure. Thus a monitoring tool for evaluating and eventually optimizing the training procedure is not available today.

We hypothesize that measuring the pressure between the skeletal muscle and the myocardium could be an important tool for evaluation of the skeletal muscle contraction force and the timing of the myostimulator-induced muscle contraction in relation to the myocardial contraction. Therefore the purpose of this study was to evaluate a newly designed pressure registration system in two established DCMP animal models. 

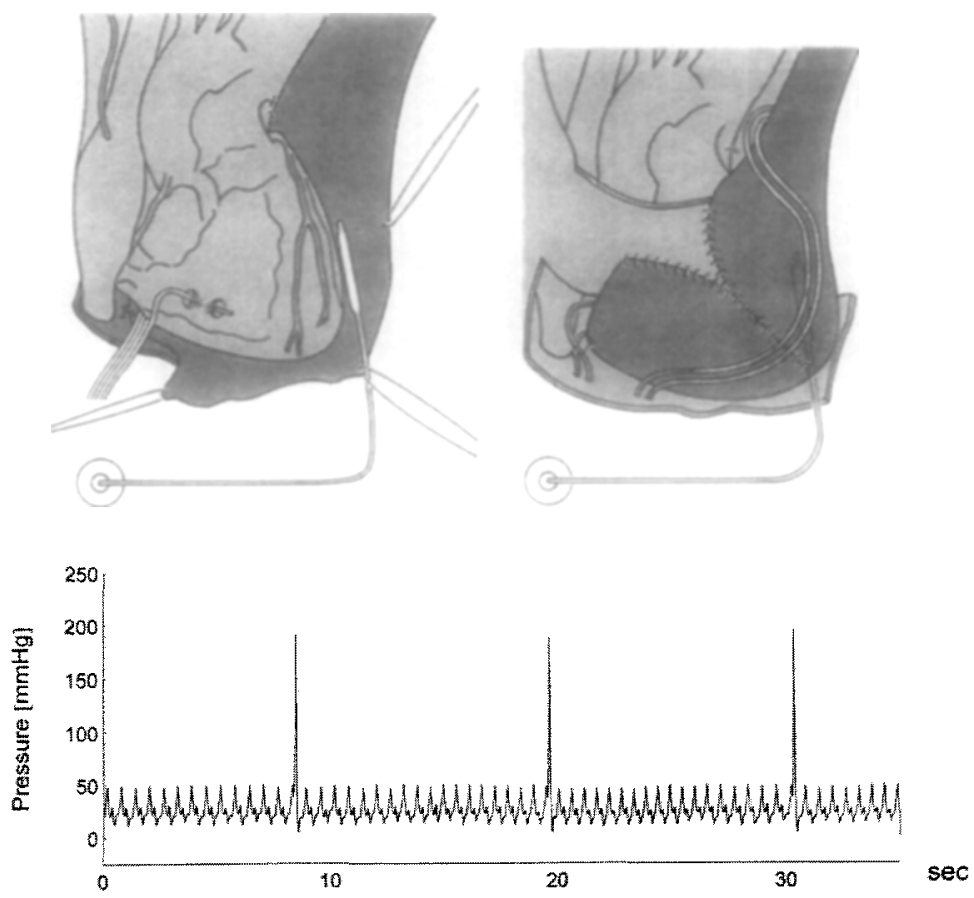

Fig. 1. Top, The cardiomyoplasty monitoring catheter, consisting of a saline solution filled balloon, a double-lumen catheter, and a vascular access port, is placed between the wrap of LD muscle and the base of the posterior wall of the left ventricle. Bottom, A typical recording from the DCMP catheter showing heart contractions with three DCMP contractions in 30 seconds.

Table I. Long-term results of animal study with a monitoring catheter for a DCMP

\begin{tabular}{ccclc}
\hline & & $\begin{array}{c}\text { Stimulation } \\
\text { mode } 2 \text { mo } \\
\text { No. }\end{array}$ & $\begin{array}{c}\text { Functional } \\
\text { state 2 and }\end{array}$ & $\begin{array}{c}\text { Morphology } \\
\text { of DCMP }\end{array}$ \\
\hline 1 & Goat & $1: 2$ & Failure & Damaged \\
2 & Goat & $1: 2$ & Failure & Damaged \\
3 & Goat & $1: 4$ & Failure & Damaged \\
4 & Goat & $1: 8$ & Contraction & Preserved \\
5 & Goat & $1: 8$ & Contraction & Preserved \\
6 & Pig & $1: 8$ & Contraction & Preserved \\
7 & Pig & $1: 8$ & Contraction & Preserved \\
\hline
\end{tabular}

\section{Material and methods}

The implantable DCMP-monitoring double-lumen catheter (Fig. 1), constructed of polyurethane, was about $45 \mathrm{~cm}$ in length and $1.3 \mathrm{~mm}$ in diameter. At the tip, a nonextensible polyurethane balloon was mounted. Its diameter was $4 \mathrm{~mm}$ and its length $30 \mathrm{~mm}$. The other end of the catheter was bifurcated: one branch entered a small vascular access port (Access Technologies, Skokie, Ill.) measuring chamber and the second branch a flexible tube that could be closed after filling. The balloon was filled with hypertonic saline solution to its maximal extent and then adjusted to atmospheric pressure. This procedure was repeated before each measurement to standardize measuring conditions.

Five adult female Boor goats weighing 38 to $45 \mathrm{~kg}$ and between 3 and 5 years old were used in this study in addition to two domestic pigs weighing 52 and $58 \mathrm{~kg}$, respectively. The goats were kept under veterinary care at the Animal Department of the Medical University of Lübeck. They were supervised by a representative of the Society for the Prevention of Cruelty to Animals from the government of Schleswig-Holstein, Kiel, Germany. The two pigs were kept under the veterinary care of Aarhus University, Denmark. The study was controlled according to methods approved by the Danish Inspectorate for Animal Experiments.

Xylazine hydrochloride 2\% (Rompun), $0.2 \mathrm{mg} / \mathrm{kg}$, and ketamine (Ketanest), $5 \mathrm{mg} / \mathrm{kg}$, were used to induce general anesthesia. After endotracheal intubation, the lungs of the animals were ventilated with oxygen, and anesthesia was maintained with continuous delivery of propofol (Disoprivane), 4 to $6 \mathrm{mg} / \mathrm{hr}$, through a central intravenous line via the internal jugular vein. Intermittent intravenous bolus delivery of a 1 to $2 \mathrm{mg} / \mathrm{kg}$ dose of ketamine was also done. There was no need to administer a muscle relaxant.

The goats and pigs were placed in the right lateral decubitus position and an oblique incision from the left axilla to the left iliac crest was made. The left latissimus dorsi muscle (LD) was dissected from its insertion and surrounding fascial attachments. Two intramuscular electrodes (Telectronics model IML-2) were inserted $6 \mathrm{~cm}$ 
1. post op. day

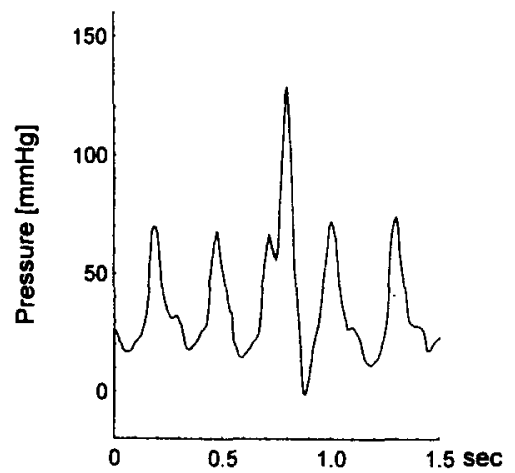

27. post op. day

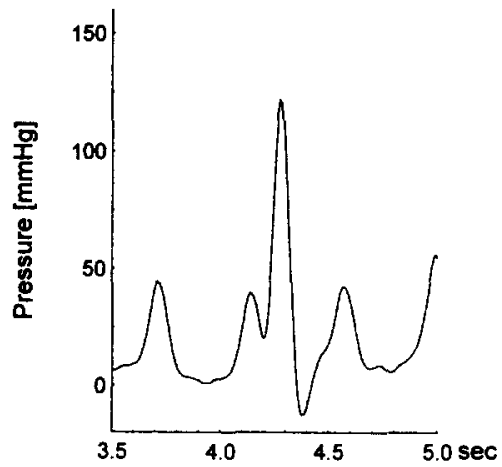

41. post op. day

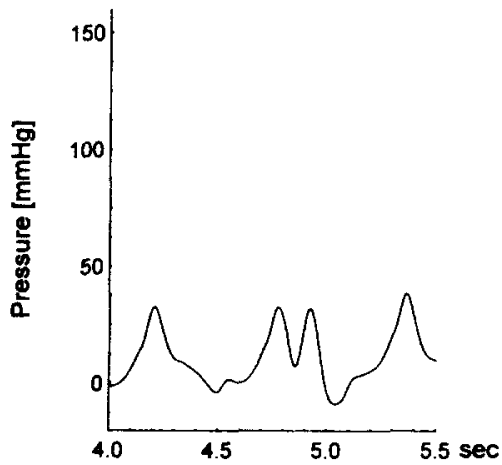

16. post op. day

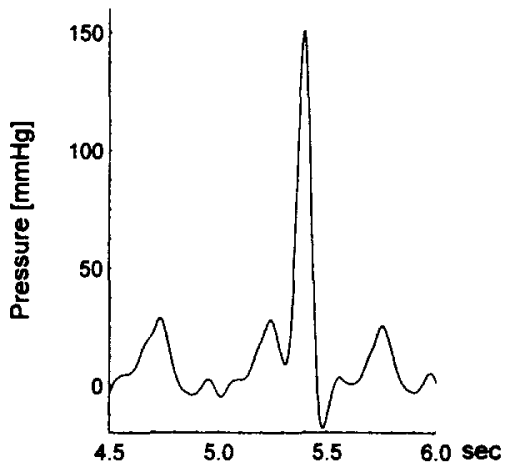

37. post op. day

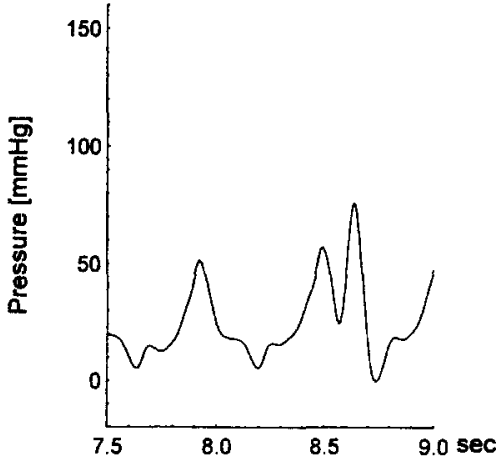

172. post op. day

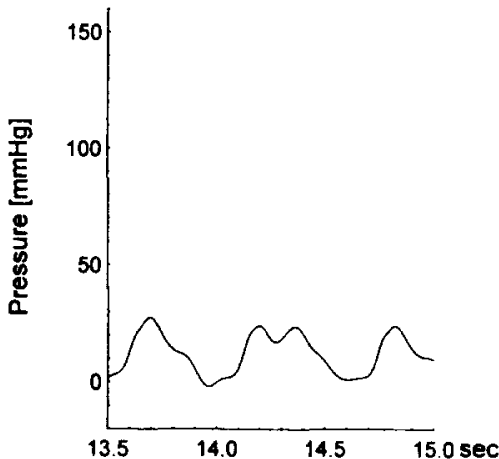

Fig. 2. Original pressure curves obtained with the DCMP monitoring catheter in goat 4 over time on postoperative days $1,16,27,37,41$, and 172 . This figure shows the variation of the pressure amplitude caused by the DCMP contraction with an increase in the maximum amplitude over the first 3 weeks, followed by a decrease. The relation between the DCMP contraction and cardiac activity can also be seen.

apart around the branches of the thoracodorsal nerve. Five to six centimeters of the third rib was resected and the LD was transferred into the thoracic cavity. The free edge of the humeral tendon was then attached to the second rib. Animals were placed in the supine position and a median sternotomy was done. The left LD cardiomyoplasty was then performed in the standard posteroan- terior fashion. The catheter was deaired by injection of saline solution. The balloon was then placed on the diaphragmatic posterior wall of the left ventricle, near the base of the ventricle. Before the DCMP wrap was completed with the use of a pericardial flap (Fig. 1, top), appropriate pressure signals of the catheter were verified. A ventricular screw-in sensing electrode (Telectronics 


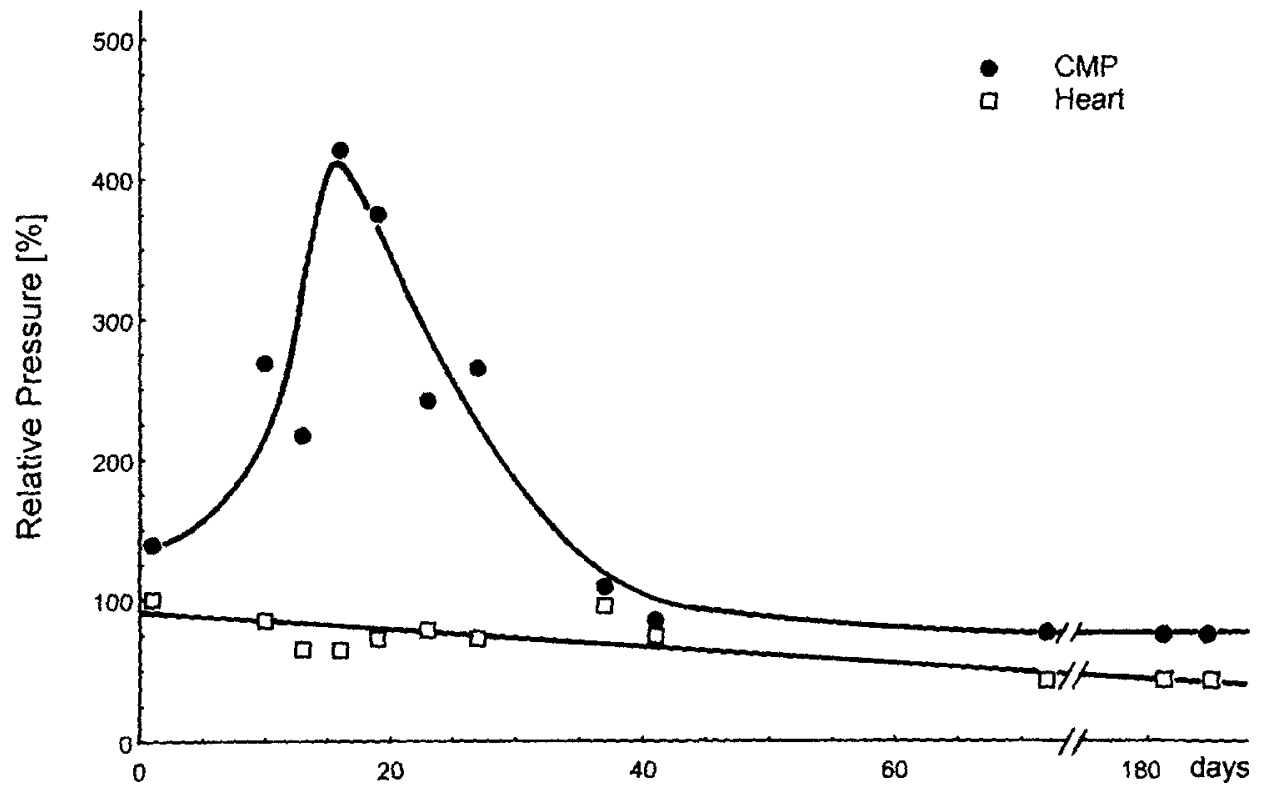

Fig. 3. Relative maximal pressure in the monitoring catheter. The upper curve shows the relative DCMP amplitude of the pressure signals (see equation 1 in the text) induced by DCMP contractions with an increase to about $421 \%$ on postoperative day 16 and a decrease to about $67.6 \%$ on postoperative day 172 . The lower curve demonstrates the relative heart amplitude as a result of cardiac motion normalized by the initial heart amplitude (see equation 2 in the text). The curve shows a decrease from $100 \%$ to $44.2 \%$. Curves are based on calculations from pressure data from goat 4 .

model 033-572) and the myoelectrodes were then connected to a myostimulator (Telectronics model 7220 ) programmed with the following stimulation variables: amplitude 2.5 volts, burst starting with 2 pulses and a frequency of $50 \mathrm{~Hz}$, a pulse width of $100 \mu \mathrm{sec}$, and a synchronization mode of 1 DCMP beat per 20 beats of the heart $(1: 20)$.

Stimulation was initiated immediately with no postoperative delay period. During the first 4 weeks after the operation the number of pulses per burst was increased from 2 to 10 (burst length 40 to $200 \mathrm{msec}$ ). In this phase the burst frequency was 1 to 20 heartbeats $(1: 20)$. In the following 8 weeks this frequency was gradually increased to $1: 2$ in goats 1 and $2,1: 4$ in goat 3 , and $1: 8$ in goats 4 and 5 and pigs 1 and 2 (Table $)$.

Pressure signals from the monitoring catheter were measured via the subcutaneous vascular access port measuring chamber pierced by a port cannula with a special bevel (Huber-Needle 20G $\times 25 \mathrm{~mm}$, Braun, Melsungen, Germany). Data collection was performed by an electromechanical pressure transducer (Hewlett Packard model $1290 \mathrm{C}$, Böblingen, Germany) and a physiologic monitor (Hewlett Packard model 78342A). All data were stored in a computer (AT 486, $33 \mathrm{MHz}$ ) and digitized with a sample frequency of $200 \mathrm{~Hz}$ with use of an $\mathrm{AD}$ converter ( $\mathrm{AD}$ 1602, 12-bit resolution, Keithley, Taunton, Mass.).

Assessment of the long-term functional state of the DCMP was based on relative change in the DCMP amplitude during an assisted cardiac cycle. This amplitude was defined as the relation between the DCMP amplitude difference normalized by the heart amplitude difference as follows:

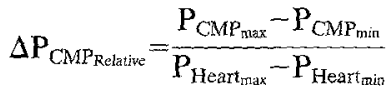

The relative change of heart amplitude over time was defined as the relation between the currently measured amplitude of the heart divided by the heart amplitude measured at the beginning of the study:

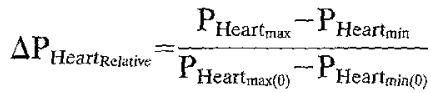

This relation is useful to document any change in signal amplitude over time, for example, change caused by encapsulation of the measuring balloon. These values were averaged from 10 cardiac cycles. Summarized results are represented by mean values and standard deviation (mean plus or minus standard deviation).

Two months after the operation, a $7 F$ conductance catheter (Cardiodynamics, Leiden, The Netherlands) was inserted in goat 4 and pig 1 . The conductance catheter was introduced by the Seldinger technique into the carotid artery and placed along the long axis of the left ventricle for real-time pressure-volume data acquisition. The mechanical signals from the cardiac cycle, as indicated by the DCMP monitoring catheter, were related to the pressurevolume data obtained by the conductance catheter to relate DCMP catheter recording to the cardiac cycle. 
Table II. Relative pressure in contracting DCMP and heart in the monitoring catheter

\begin{tabular}{lcccc}
\hline & $\begin{array}{c}\text { Max. relative pressure, } \\
\text { day } 24 \pm 6.1 \\
n\end{array}$ & $\begin{array}{c}\text { End-stage pressure, } \\
\text { day 174.6 } \\
n\end{array}$ & $n$ & $(\%)$ \\
\hline DCMP & 7 & $336.2 \pm 69.4$ & 4 & $59.8 \pm 9.7$ \\
Heart & 7 & $75.2 \pm 9.1$ & 7 & $44.9 \pm 5.1$ \\
\hline
\end{tabular}

Histologic and histochemical examinations of the transposed skeletal muscle wrap were performed to compare functional results from the DCMP monitoring catheter with morphologic findings. Biopsy specimens were taken from the region of the $\mathrm{LD}$ muscle covering the posterior heart base. Three were taken for histologic evaluation and three for histochemical analyses. The pedicle of the LD muscle was not examined. Samples for the histologic examination were carefully mounted in $5 \times 10 \mathrm{~mm}$ blocks and fixed in $4 \%$ buffered formaldehyde solution. Samples for adenosinetriphosphatase (ATPase) staining were quickly frozen in isopentane cooled in liquid nitrogen at $-160^{\circ} \mathrm{C}$. Microtome sectioning ( $8 \mu \mathrm{m}$ thickness) was performed in a cryostat. Histologic evaluation was done after hematoxylin-eosin staining and histochemical analyses for fiber type were performed by ATPase staining at $\mathrm{pH} 4.35$ according to the method of Brooke and Kaiser., 10

\section{Results}

High-quality pressure signals as a result of cardiac motion and DCMP contraction were obtained throughout training (Fig. 1). Pressure signals via the DCMP catheter were recorded several times during the observation period (Fig. 2). In four of seven animals, signals from the contracting DCMP were detected throughout the entire study period of $174.6 \pm 13.1$ days. In goats 1,2 , and 3 , in all of whom a final synchronization mode of $1: 2$ and $1: 4$ was reached, no pressure signals as a result of a DCMP contraction were observable after postoperative week 8 . In goats 4 and 5 and the two pigs, all of whom underwent a final synchronization mode of $1: 8$, no muscle failure of the examined LD muscle wrap was observed up to 6 months.

The amplitude of pressure signals from the contracting muscle wrap and the heart indicated characteristic changes over time. The maximum pressure amplitude associated with DCMP contraction increased in the first 2 to 3 weeks to a maximum of $126.3 \pm 33 \mathrm{~mm} \mathrm{Hg}$ on day $24 \pm 6.1(n=7)$ and then decreased within postoperative week 6 , leading to a steady state of $22 \pm 3.4 \mathrm{~mm} \mathrm{Hg}(n=4)$. The maximal pressure amplitude from the heart on the first postoperative day of $37.9 \pm 6.9 \mathrm{~mm} \mathrm{Hg}$ exhibited a decline to $22.5 \pm 3.9 \mathrm{~mm} \mathrm{Hg}$ at the end of the training.

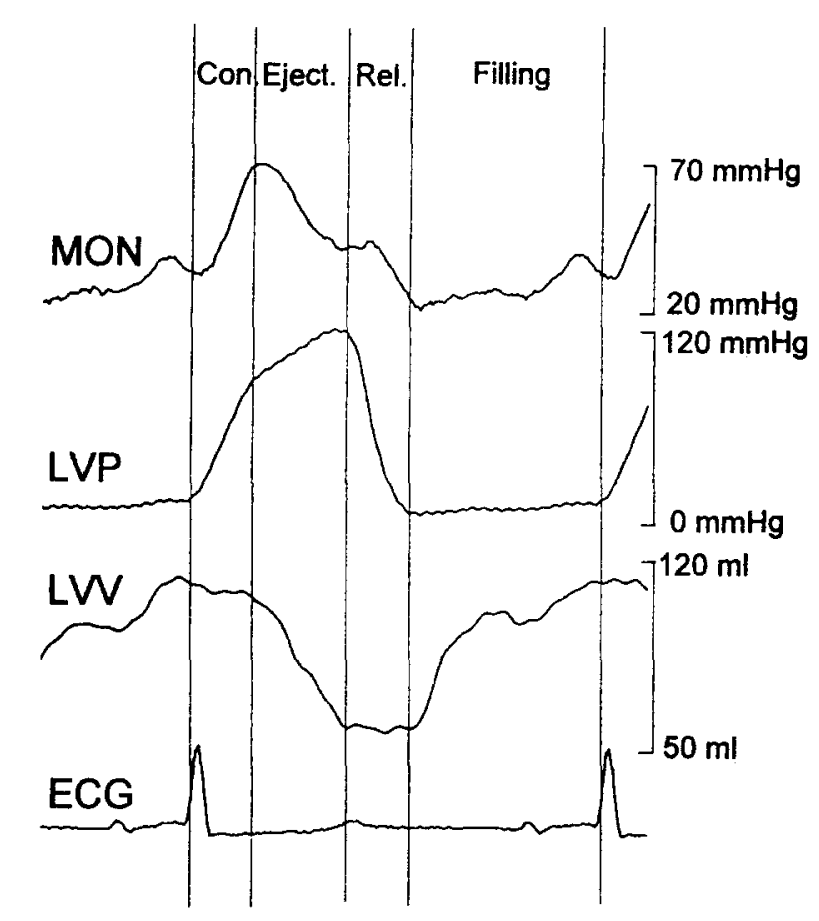

Fig. 4. Heart signals (MON) recorded via the DCMP monitoring catheter are related to the heart cycle with the use of left ventricular pressure ( $L V P$ ) and left ventricular volume $(L V V)$ measured via a conductance catheter placed in the left ventricle (unassisted beat). From the monitored signals of the heart (MON) contraction (Con), ejection (Eject.), relaxation (Rel.), and filling phases can be distinguished.

An example of changes in pressure amplitude during DCMP and the heart contractions is shown in Figs. 2 and 3. The curves in Fig. 3 depict the relative pressure development. The amplitude of the relative DCMP signal showed an increase to a maximum of $421 \%$, followed by a decline to a steady state of $67.6 \%$. The relative pressure amplitude from the heart signal decreased to a steady state of 44.2\%. Maximal relative pressure amplitudes of DCMP signals in all animals showed an increase up to a maximum of $336.2 \% \pm 69.4 \%$ on day $24 \pm 6.1$ $(n=7)$ and decreased to a steady state with an end-stage value of $59.8 \% \pm 9.7 \%$. The relative heart signal declined from $100 \%$ initially (definition) to $44.9 \% \pm 5.1 \%$ (Table II).

Simultaneously recorded pressure signals from the DCMP monitoring catheter and the pressurevolume signals from the conductance catheter (pig 1) are illustrated in Fig. 4 along with the associated electrocardiogram. Cardiac activity monitored by the DCMP catheter showed an ascending and a 


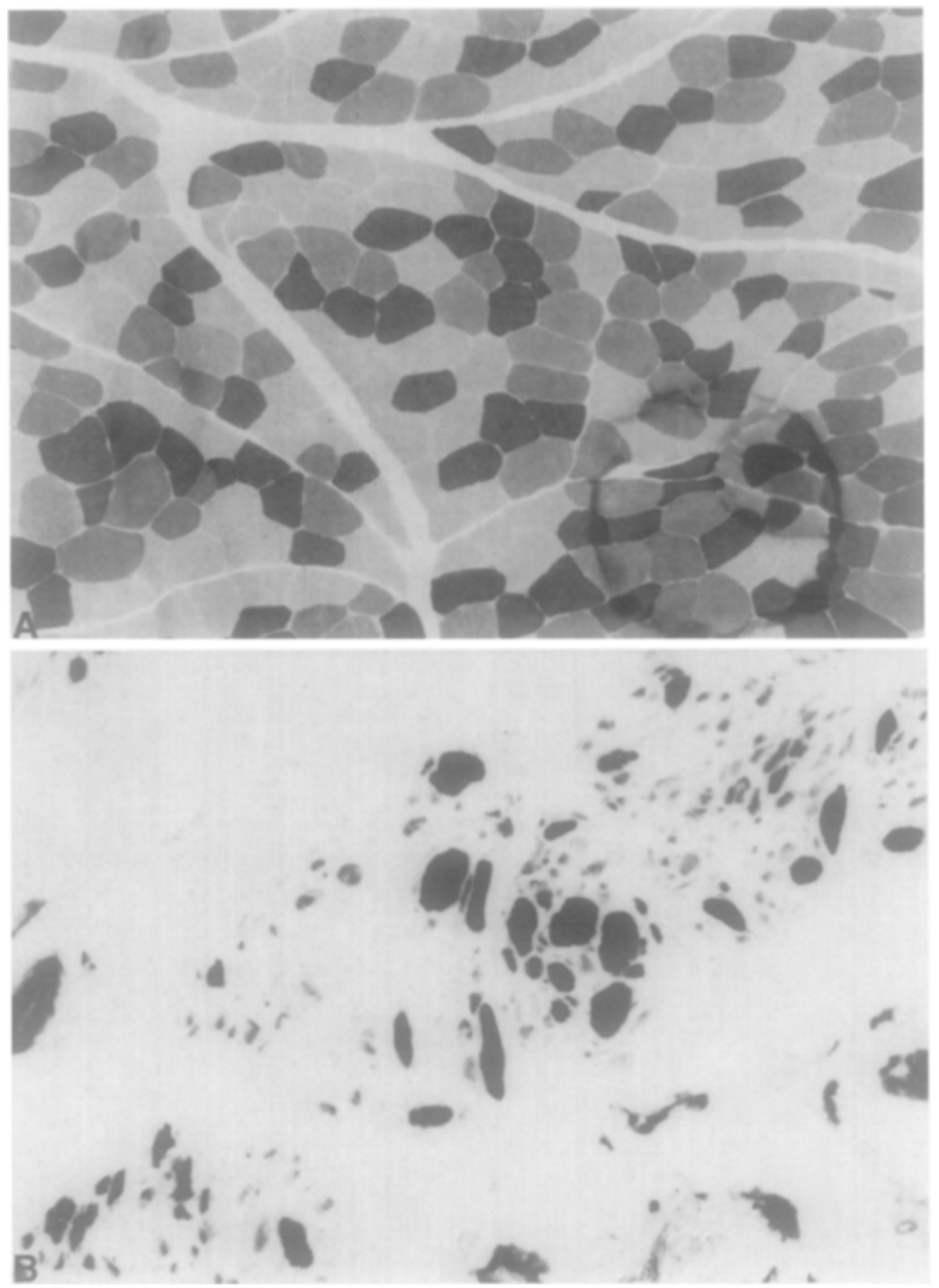

Fig. 5. A, Cross section of untrained normal LD muscle from goat 1, visualized by ATPase staining: type I fibers are dark, type IIa fibers white, and type IIb fibers gray (staining method of Brooke and Kaiser ${ }^{9}$, original magnification $\times 25$ ). B, Muscle tissue of the DCMP from goat 1 after 6 months of electrical stimulation in 1:2 mode. Only a few type I muscle fibers are left. This finding explains the loss of the DCMP signal transferred by the monitoring catheter after postoperative week 8 (staining method of Brooke and Kaiser ${ }^{9}, 10$; original magnification $\times 25$ ).

descending curve (Fig. 4). The descending part can be divided into an ejection segment and a relaxation segment.

After the balloon of the monitoring catheter was deflated, the entire catheter was extracted without any complications in all animals at the end of the study.

At autopsy (Table I), the muscle wraps showed impressive morphologic differences. The LD muscle wrapped around the heart in goats 1,2, and 3 was thin and fibrotic (Fig. 5, B), compared with that in goats 4 and 5 (Fig. 6, $B$ ). The muscle wrap in the two pigs was well preserved (Fig. 7, $B$ ), like that in goats 4 and 5.

Histologic and histochemical examination findings in goats 1,2 , and 3 , with a final synchronization mode of $1: 2$ or 1:4, differed from those of goats 4 and 5 and the pigs, with a final stimulation mode of 1:8. In goats 1, 2, and 3 most muscle fibers were damaged and impressive replacement by fibrotic 

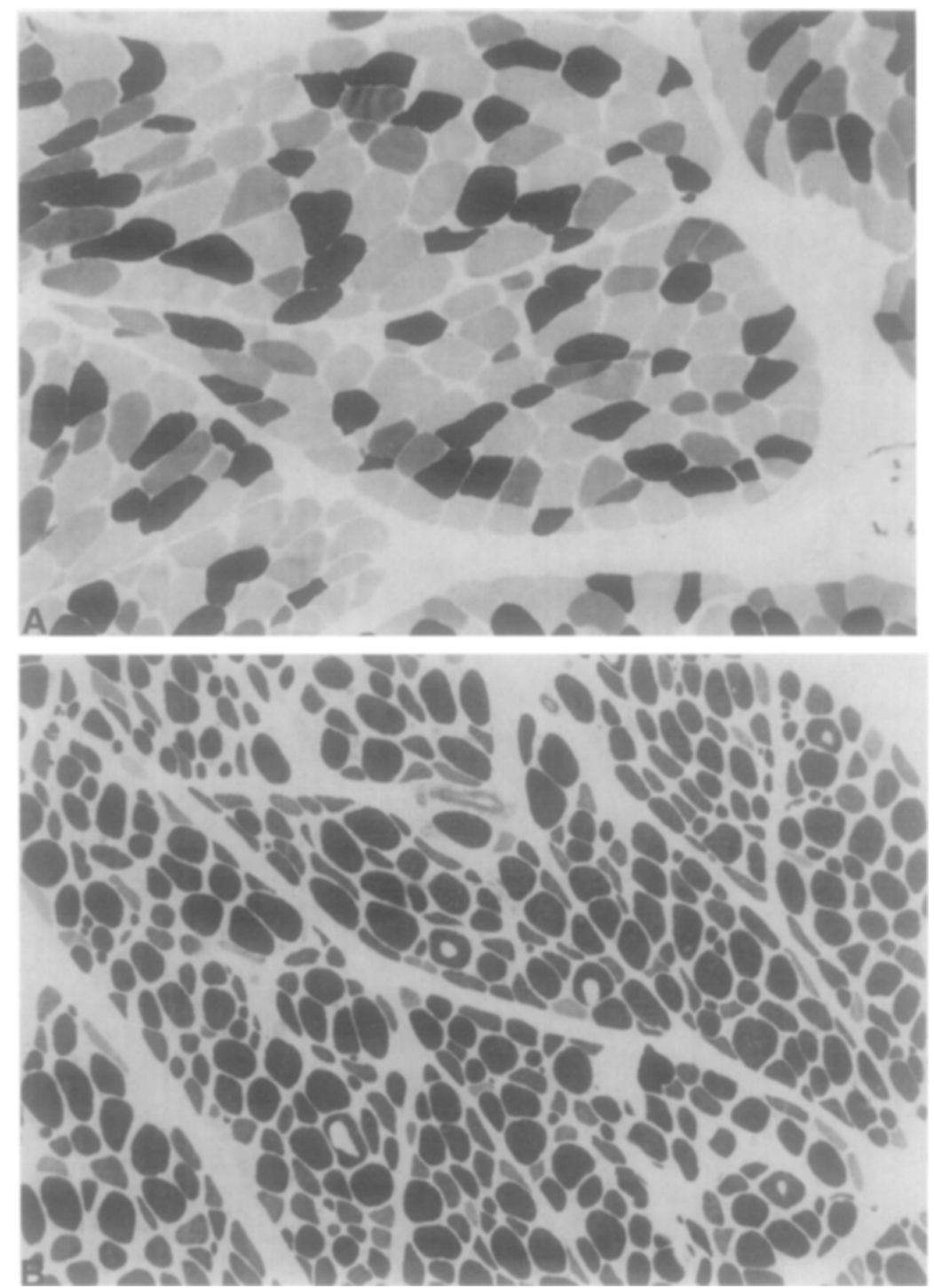

Fig. 6. A, Normal muscle fibers without cell damage from the untrained LD muscle of goat 4 (staining method of Brooke and Kaiser ${ }^{9}, 10$; original magnification $\times 25$ ). B, DCMP muscle tissue with solely type I fibers from goat 4 with an end-stage stimulation of 1:8 showing no muscular damage. The monitoring catheter transferred DCMP signals over 6 months up to study termination (staining method of Brooke and Kaiser $^{9,10}$; original magnification $\times 25$ ).

and fatty tissue was observed (Fig. 5, B). Cross sections of the nonstimulated LD muscles of the goats (Figs. 5, $A$, and $6, A$ ) and the pigs (Fig. 7, $A$ ) were visualized by an ATPase staining for fiber typing by the method of Brooke and Kaiser ${ }^{9,10}$ at a $\mathrm{pH}$ of 4.53. Type I fibers were dark, type IIa white, and type IIb gray. (Type I are slow twitch and fatigue resistant and type II are fast twitch but fatiguable muscle fibers.) Nonstimulated muscle tissue consisted of a mixture of type I and type II fibers as shown in Figs. $5, A, 6, A$, and $7, A$. After a stimulation period of about 6 months, the LD muscles in goats 1,2, and 3, stimulated at 1:2 and $1: 4$, contained only a few fibers (type I), surrounded by connective and fatty tissue (Fig. 5, B). Most muscular tissue (type I fiber) was maintained in goats 4 and 5 and the two pigs, all with a final synchronization mode of $1: 8$ (Figs. $6, B$, and $7, B$ ). In these four animals the DCMP signals were detected up to the end of study. In this study the loss of the 

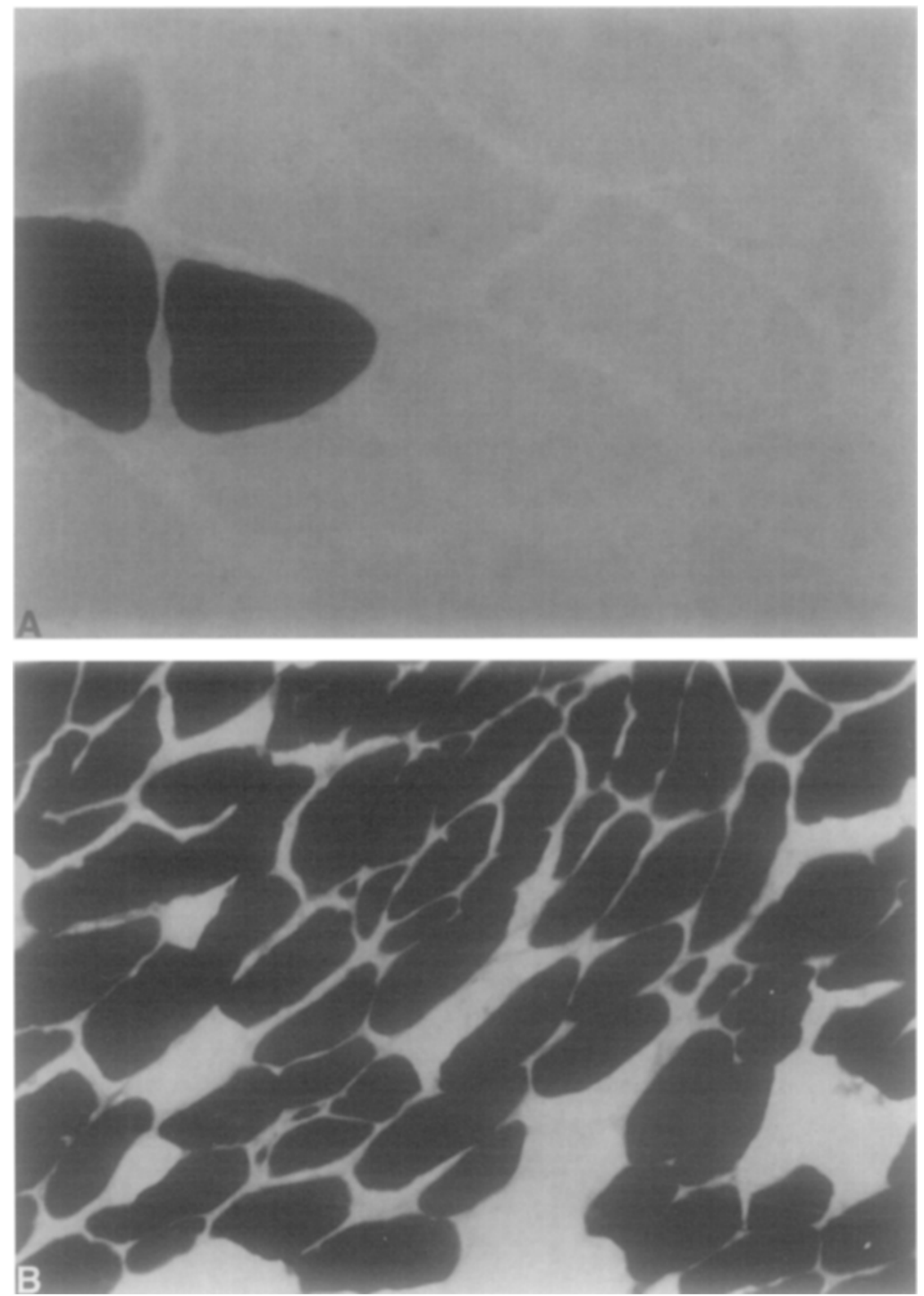

Fig. 7. A, Normal muscle tissue from the LD muscle from a pig (staining method of Brooke and Kaiser $^{9,}{ }^{10}$; $\mathrm{pH} 4.35$; original magnification $\times 40$ ). B, Stimulated muscle tissue of the DCMP from a pig after 6 months with an end-stage synchronization mode of 1:8 showed type I fibers with minimal muscle damage. The monitoring catheter transferred clear DCMP pressure signals up to study termination at 6 months after operation (staining method of Brooke and Kaiser ${ }^{9}{ }^{10}$; $\mathrm{pH} 4.35$; original magnification $\times 40$ ).

DCMP signals was found to correlate with severe morphologic deterioration of the muscle tissue.

\section{Discussion}

Investigations with a fluid-filled measuring balloon interposed between the myocardium and LD muscle were reported previously to evaluate the transmural myocardial pressure after a DCMP procedure. ${ }^{11}$ The pressure difference between the left ventricular cavity and the balloon was defined as transmural myocardial pressure. In that short-term study, a significant decrease in transmural myocardial pressure as an effect of DCMP was demonstrated. The purpose of our study, however, was to monitor the functional state of a DCMP over 6 months.

All seven monitoring catheters reported here showed an excellent transfer of pressure signals caused by heart activity from the beginning to the end of the study and from the DCMP when it was 
contracting well. The use of the hyperosmotic saline solution in the inner lumen of the catheter was intended to avoid a major volume loss. Nevertheless small quantities of volume had to be replaced before each pressure measurement to achieve comparable filling conditions.

Signals from the DCMP catheter provided information about the synergically contracting muscle in the entire area of the posterior wrap. The balloon was placed in the region where the DCMP covered the largest circumference of the heart. In the classic DCMP using the left LD muscle, the posterior wall of the left ventricle is always covered with the best textured part of the LD muscle. Therefore a balloon of $30 \mathrm{~mm}$ in length in that position gave representative information on the functional state of this consequential part of the muscle wrap. Perhaps a longer balloon might integrate a larger area of contracting tissue for a more representative evaluation of DCMP function.

The increase in amplitude within the first 3 weeks after operation might have been caused mostly by the increasing number of pulses per burst by our stimulation pattern and partly by the postoperative recovery of the muscle. The decrease in amplitude of the DCMP signals might have been caused by the loss in power, which was about one eighth of the original state, as is well-known and documented. ${ }^{12}$ This was a result of fiber transformation from powerful type II fibers to less powerful but fatigueresistant type I fibers (Figs. 5, $B, 6, B$, and $7, B$ ).

Connective tissue reaction to the foreign material of the measuring balloon is supposed to have an influence on the measured signals. This was indicated by the relative heart amplitude, which decreased over time to $59.5 \% \pm 13.9 \%$ of its original value. Of course, this decrease may be a dampening caused by encapsulation, but this effect could be overcome by the relative pressure calculation in which both pressure values (DCMP and heart) were influenced by the same connective tissue.

Muscle damage described after a stimulation mode of $1: 2$ and $1: 4$ was combined with an increase in connective and fatty tissue between the remaining type I fibers. These morphologic findings might be a result of extreme overstimulation with muscle damage and neural degeneration. Nevertheless, an ischemic origin of this muscular destruction cannot be excluded. A 1:8 mode was associated with maintained function and preserved muscle tissue. These findings are related to good clinical outcome in 14 patients with DCMP after 51/2 years with a $1: 6$
Table III. Uses for a DCMP monitoring catheter

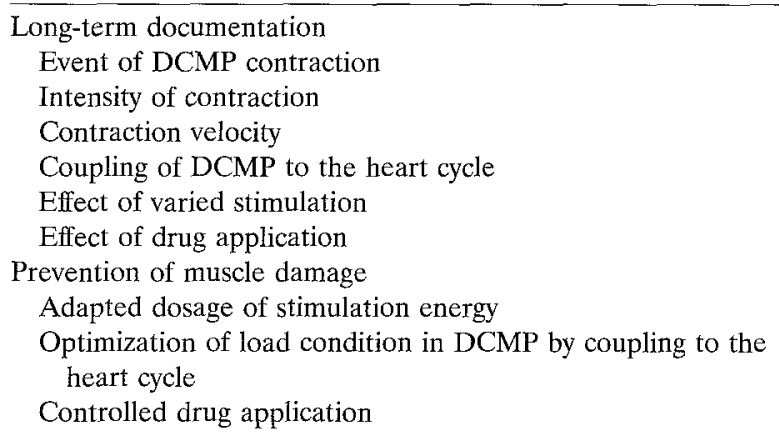

stimulation ratio. ${ }^{13}$ We speculate that muscle damage will be key to long-term successful therapy. Further studies need to be undertaken to verify this finding under conditions that control for other possible causes of muscle damage.

Mechanical activity of the base of the posterior wall of the left ventricle (Fig. 4, MON) consisted of an ascending curve, a peak, and a descending part. The descending part was characterized by a small peak, which separated an ejection part from a relaxation section as shown in the measurements of the conductance catheter. These signals of the DCMP catheter allow synchronization of the DCMP with the heart cycle. If the ejection fraction of the heart should be improved, stimulation can be adjusted to induce contraction during the ejection phase. If a girdling or a remodeling is needed, ${ }^{8}$ stimulation during the relaxation part might be conceivable but would need to be applied in a way that does not impair diastolic filling. It could be worthwhile to consider a "walk behind" contraction, which would mimic dynamic training during electrical conditioning, shown in skeletal muscle ventricles, to maintain or increase muscle power. ${ }^{14,15}$

Clinical complications of applying an extravascular catheter should be low. Covering the catheter near the measuring chamber with a piece of Dacron felt for better integration with the tissue could prevent spreading of infection from skin to the heart.

Whereas the conductance catheter enables direct analysis of myocardial improvement, ${ }^{16,17}$ this monitoring catheter can be applied to verify the contractile function of the $\mathrm{LD}$ muscle and the relative intensity of its contraction. Even contraction velocity can be documented by the slope of the DCMP contraction curve (not evaluated in this study). The coupling of the DCMP to the heart cycle can be observed, as well as the effect of varying stimulation 
parameters. Therefore this catheter could be useful in optimizing stimulation patterns; muscle damage can be prevented by adjusting the stimulation energy and by adjusting the loading conditions of DCMP through careful timing of the contraction during the heart cycle. Any benefit of drugs administered to strengthen the LD muscle could also be monitored. In our opinion, this monitoring catheter could be helpful in improving the outcome of the surgical treatment of heart failure with DCMP (Table III).

We thank Mrs. Kerstin Lünsmann for her excellent care of the goats.

\section{REFERENCES}

1. Carpentier A, Chachques IC. Myocardial substitution with a stimulated skeletal muscle: first successful clinical case [letter]. Lancet 1985;8440:1267.

2. Chiu RC-J. Dynamic cardiomyoplasty: an overview. Pacing Clin Electrophysiol 1991;14:577-84.

3. Delahaye F, Jegaden $O$, Montagna $P$, Desseigne $P$, Blanc $P$, Vedrinne $C$. Latissimus dorsi cardiomyoplasty in severe congestive heart failure: the Lyon experience. J Cardiac Surg 1991;6(suppl. 6.1):106-12.

4. Jatene AD, Moreira LFP, Stolf NAG, et al. Left ventricular function changes after cardiomyoplasty in patients with dilated cardiomyopathy. J Thorac Cardiovasc Surg 1991;102:132-9.

5. Magovern GJ, Heckler FR, Park SB, et al. Paced skeletal muscle for dynamic cardiomyoplasty. Ann Thorac Surg 1988; 45:614-9.

6. Moreira LF, Stolf NA, Bocchi EA, et al. Latissimus dorsi cardiomyoplasty in the treatment of patients with dilated cardiomyopathy. Circulation 1991;82:257-62.

7. Schreuder JJ, van der Veen FH, van der Velde ET, et al.
Beat-to-beat analysis of left ventricular pressure-volume relation and stroke volume by conductance catheter and aortic modelflow in cardiomyoplasty patients. Circulation 1995;91: 2010-7.

8. Kass DA, Baughman KL, Pak PH, et al. Reverse remodeling from cardiomyoplasty in human heart failure: external constraint versus active assist. Circulation 1995;91: 2314-8.

9. Brooke MH, Kaiser KK. Some comments on the histochemical characterization of muscle adenosine triphosphatase. J Histochem Cytochem 1969;17:431-9.

10. Brooke MH, Kaiser KK. Muscle fiber types: how many and what kind? Arch Neurol 1970;23:369-79.

11. Chen FY, Aklog L, deGuzman BJ, et al. New technique measures decreased transmural myocardial pressure in cardiomyoplasty. Ann Thorac Surg 1995;60:1678-82.

12. Salmons S, Jarvis JC. Cardiac assistance from skeletal muscle: a critical appraisal of the various approaches. Br Heart J 1992;68:333-8.

13. Chekanov V. Dynamic cardiomyoplasty at 51/2 years: Russian clinical experience. J Thorac Cardiovasc Surg 1995;109:18990.

14. Guldner NW, Eichstädt HC, Klapproth P, et al. Dynamic training of skeletal muscle ventricles: a method to increase muscular power for cardiac assistant. Circulation 1994;89: 1032-40.

15. Guldner NW, Tilmans MHJ, DeHaan H, Ruck K, Bressers $\mathrm{H}$, Messmer BJ. Development and training of skeletal muscle ventricles with low preload. J Card Surg 1991;6: 175-83.

16. Baan J, van der Velde ET, De Bruin H. Continuous measurement of left ventricular volume in animals and men by conductance catheter. Circulation 1984;70:812-23.

17. Kass DA, Beyar R, Lankford E, Heard M, Maughan WL, Sagwa K. Influence of contractile state on the curvilinearity of the in situ end-systolic pressure-volume relations. CircuIation 1994;79:167-78. 